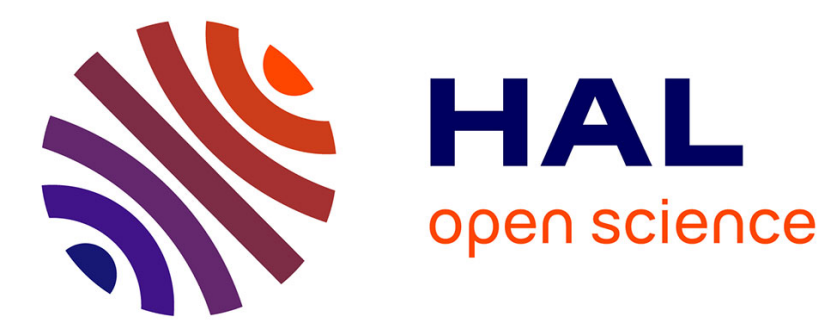

\title{
Le francien (1815-1914): la linguistique au service de la patrie
}

Gabriel Bergounioux

\section{To cite this version:}

Gabriel Bergounioux. Le francien (1815-1914) : la linguistique au service de la patrie. Mots Pluriels, 1989, 19 (1), pp.23 - 40. 10.3406/mots.1989.1463 . halshs-01820552

\section{HAL Id: halshs-01820552 \\ https://shs.hal.science/halshs-01820552}

Submitted on 21 Jun 2018

HAL is a multi-disciplinary open access archive for the deposit and dissemination of scientific research documents, whether they are published or not. The documents may come from teaching and research institutions in France or abroad, or from public or private research centers.
L'archive ouverte pluridisciplinaire HAL, est destinée au dépôt et à la diffusion de documents scientifiques de niveau recherche, publiés ou non, émanant des établissements d'enseignement et de recherche français ou étrangers, des laboratoires publics ou privés. 


\section{Le francien (1815-1914) : la linguistique au service de la patrie}

In: Mots, juin 1989, №19. pp. 23-40.

\section{Resumen}

EL «FRANCIEN», LINGUISTICA AL SERVICIO DE LA PATRIA EI « francien », dialecto de l'lle-de-France, representaria el francés « standard » de la Edad Media, pero de hecho este concepto corresponde a una esperanza jacobina de las clases dominantes de la Tercera República, retomada por unos universitarios en busca de una legitimidad y de una estabilización del mercado de la linguistica bajo la férula de G. Paris. Instrumenta operative y sintoma de una politica de la ciencia dedicada a una historia apologética del francés, el « francien » parece ser una ficción científica.

\section{Abstract}

THE «FRANCIEN » : LINGUISTICS IN THE SERVICE OF HOMELAND The « francien » is the dialect of lle-de-France, it would have represented the standard French in the Middle Ages, but, in fact, this concept corresponds to the jacobin views of the Third Republic ruling classes, converging with university scholars longing for social recognition, new wording and linguistic stabilization. Devoted to the praise of the french language, the « francien » seems to be a scientific fiction.

\section{Résumé}

LE FRANCIEN (1815-1914) : LA LINGUISTIQUE AU SERVICE DE LA PATRIE Le francien, dialecte de I'lle-de-France, représenterait le français "standard » du Moyen Age, mais ce concept correspond en fait à une attente jacobine des classes dominantes de la Troisième République, relayée par des universitaires en quête de légitimité, et à une stabilisation du marché de la linguistique sous la férule de G. Paris. Outil opératoire et symptôme d'une politique de la science vouée à une histoire apologétique du français, le « francien » relève de la fiction scientifique.

Citer ce document / Cite this document :

Bergounioux Gabriel. Le francien (1815-1914) : la linguistique au service de la patrie. In: Mots, juin 1989, №19. pp. 23-40.

doi : $10.3406 /$ mots. 1989.1463

http://www.persee.fr/web/revues/home/prescript/article/mots_0243-6450_1989_num_19_1_1463 
Gabriel BERGOUNIOUX

Université d'Orléans

\section{Le francien (1815 - 1914) : la linguistique au service de la patrie}

La première attestation de francien remonte à $1889^{1}$. Loin de représenter un progrès dans les reconstructions successives de l'ancien français, il ne s'agit que d'une nouvelle désignation pour le dialecte de l'lle-de-France dont la postulation est indissociable des controverses dialectologiques, en France, au 19e siècle. Cette substitution révèle en revanche par quel infléchissement de leurs conceptions linguistiques les romanistes rejoignirent les sollicitations des cercles de production intellectuelle de la Troisième République triomphante, ceux dont $C$. Nicolet a rassemblé l'œuvre sous le vocable d'« idée républicaine ».

Un récapitulatif des enjeux dialectologiques avant et après la création de la revue Romania (1872) doit permettre de comprendre les transformations, scientifiques et sociales, du statut du parler parisien dont l'existence n'est établie que par une dénomination, francien, qui marque l'achèvement d'une démarche savante, à défaut d'être scientifique.

1. L'histoire de «francien » a fait l'objet d'excellentes monographies de A. Henri, M-R. Simoni-Aurembou et, de manière particulièrement détaillée, de J. Chaurand dans les Mélanges Robert Loriot («Pour l'histoire du mot francien", Fontaine-lès-Dijon, ABDO (Association bourguignonne de dialectologie et d'onomastique), 1983, p. 91-99). Nous sommes redevables à Guilaine Brun, Bernard Cerquiglini, Jean-Claude Chevalier, Simone Delesalle, Pierre Encrevé et tout particulièrement Marie-Rose Simoni-Aurembou de nombreuses et profitables remarques. Qu'ils en soient remerciés. 


\section{Apparition de l'idée de dialecte (1830-1870) : de Fallot à Littré}

Alors que les enquêtes de Grégoire et de Coquebert de Montbret effectuaient une première reconnaissance des parlers en France, leur identité reste indéfinie : s'agit-il de patois, d'idiomes, de langages ? Girault-Duvivier signale que « dialecte " n'est « en usage que parmi les gens de lettres, et seulement quand il s'agit de grec ». L'université demeure réfractaire à ces " corruptions » du bon langage comme à celle des états anciens du français. A la même époque, en Allemagne, Diez rédige la première grammaire comparée et historique des langues romanes; en Suisse, Orell étudie la morphologie de l'ancien français, tous deux insistant sur le témoignage des patois, sans rencontrer d'écho en France jusqu'à l'étude de G. Fallot.

Gustave Fallot (1807-1836) est l'un de ces savants dont l'œuvre inachevée scelle la possibilité d'un bouleversement du champ de la linguistique. Chartiste, érudit, parcourant les bibliothèques, il a découvert Orell et Diez dans le texte. D'une sensibilité proche du romantisme, il est fasciné par l'œuvre, philologique et littéraire des frères Grimm dont il rêve de produire l'équivalent en France. Ses Recherches sur les formes grammaticales de la langue française et de ses dialectes au XIIIe siècle (1839, posthume) rompent d'un même mouvement avec la frileuse tradition philologique française et avec une conception unitaire de l'ancien français, puisqu'il précise presque immédiatement :

"Les anciennes provinces de France ont donc eu d'abord un langage identique au fond, mais différent dans le détail. Lorsque l'on a commencé d'écrire, dans chacune de ces provinces, en langage vulgaire, on n'a pu écrire que dans l'idiome, ou, pour mieux dire, dans le dialecte de la province. Ce n'est que plus tard que la langue française proprement dite est née du mélange et de la fusion de ces différents dialectes; et ce n'est que bien plus tard encore qu'elle les a fait déchoir tous du rang de langages écrits, et relégués au rang de patois ${ }^{\prime}$.

Les subdivisions dialectales sont ramenées à trois : « Je ne crois pas, après bien des observations et des comparaisons, qu'il soit

1. Gustave Fallot, Recherches sur les formes grammaticales de la langue française et de ses dialectes du 13 e siècle, Paris, Imprimerie royale, 1839, p. 10. 
nécessaire de diviser le vieux langage français en plus de trois dialectes principaux/.../, normand, picard et bourguignon " ${ }^{1}$.

Les dix premières pages du livre de G. Fallot postulent que :

1) l'ancien français désigne l'ensemble des trois dialectes de la moitié nord de la France (le sud étant le domaine du provençal); 2) le parler de l'Ile-de-France relève du bourguignon ; 3) seul le traitement des voyelles et consonnes permet de déterminer les frontières dialectales ; 4) l'étude du phonétisme requiert le recours aux textes du moyen-âge (il en cite trois pour le sous-dialecte de l'Ile-de-France).

Les théories de G. Fallot déclenchent des critiques plus ou moins aiguës de la part de ses successeurs. F. Wey soutient les droits du celtique, F.Génin récuse l'existence même des dialectes. J.-J. Ampère, plus au fait des thèses de la romanistique, discute thèse à thèse, déclarant notamment :

«Il y a d'abord quelque chose d'étrange à donner le nom de bourguignon au français parlé dans l'Ile-de-France et au bord de la Loire, c'est-à-dire au français qui est devenu la langue de Paris et l'idiome littéraire de notre pays. Il est bien certain que, dès le XIIe siècle, on considérait le langage de l'Ile-de-France comme le meilleur $»^{2}$.

Encore J.-J. Ampère s'empresse-t-il de préciser que le dialecte de l'Ile-de-France est proche du bourguignon (il ne peut citer qu'un trait phonétique qui les distingue) avant de conclure sur la modicité des différences dialectales. Il aura néanmoins marqué une étape : désormais le français a quatre dialectes principaux, comme en témoigneraient les écrivains du Moyen Age, notamment "Coenes de Béthune", et les patois dont G. Fallot n'avait pas fait usage. Dans les années 1850, Littré en fixe la formule, ainsi condensée dans la préface de son Dictionnaire:

« Pourquoi des dialectes et non pas des patois ? C'est qu'alors l'unité de langage et de littérature n'existait pas/.../A cette haute époque, ce sont les littératures de la Normandie, de la Picardie et de l'Ile-deFrance qui ont la primauté par le nombre et la qualité des auvres. Quand le quatorzième siècle finit/.../c'est à ce moment que les dialectes cessent d'exister en France ; les patois en prennent la place. Ainsi l'on définira le patois un dialecte qui, n'ayant plus de culture littéraire, sert seulement aux usages de la vie commune".

1. Ibid., p. 14

2. Jean-Jacques Ampère, Formation de la langue française, Paris, Didier, 1869, p. 69 (1re édition, 1841). 
La position éminente de Littré, autant que l'équilibre qu'il parvient à garder dans son écriture entre le désenchantement propre au discours savant et l'appropriation au public lettré, ancrent, dans l'opinion des cercles intellectuels et politiques, l'assertion, sans justification, qu'il existe un dialecte spécifique de l'Ile-de-France.

\section{Les romanistes contre les dialectes}

\section{Avec Littré, contre Ascoli}

A la fin des années 1860, Michel Bréal (né en 1832), Gaston Paris (né en 1839) et Paul Meyer (né en 1840) parviennent à investir l'enseignement supérieur et à dominer le champ de la linguistique en France en recourant aux théories comparatistes allemandes ${ }^{1}$. G. Paris et P. Meyer, élèves de F. Diez, institutionnalisent la romanistique française, qu'ils dotent d'une revue prestigieuse, Romania (1872), et s'abritent derrière la caution nationale de certains de leurs prédécesseurs afin de contourner l'argumentation chauvine en fait anti-allemande, utilisée par les universitaires français que la redéfinition des contenus scientifiques dépouille de leur autorité institutionnelle.

Vers 1875, une acide polémique oppose P. Meyer à G.-I. Ascoli, un autre élève de $F$. Diez, le fondateur de l'école italienne de romanistique; l'enjeu : les divisions linguistiques de la France romane ( il en résultera le concept de «franco-provençal »). Ce n'est pas le lieu de reconstituer les pièces et les raisons de ce débat, seule la conclusion de P. Meyer nous intéresse : il n'y a pas de dialectes dans le domaine gallo-roman (G.-I. Ascoli restera sur des positions diamétralement opposées). Pour les deux directeurs de Romania, la notion même de dialecte devient un artefact du linguiste, démenti par le travail d'enquête sur le terrain qui ne livre qu'un continuum d'infimes variations dont les limites ne se superposent jamais, quelle que soit la frontière que l'on privilégie. Position radicale, position désespérée aussi, comme en témoigne, matériellement, sa publication : trois pages dans la livraison de juillet 1875 de Romania. A notre connaissance,

1. Cf. notre article sur « La science du langage en France de 1870 à 1885 : du marché civil au marché étatique », Langue française, 63, septembre 1984, p. 740. 
P. Meyer n'a jamais collecté d'informations auprès de locuteurs patoisants, ce qu'ont fait les provençalistes - Bringuier et Ch. de Tourtoulon, par exemple - qui souhaitaient défendre la spécificité de la langue d'oc. Force est de constater que cette thèse unitaire rejoint l'idéal jacobin des républicains. En abolissant la coupure oc/oïl, P. Meyer rend à la France son unité imaginaire, réduisant le problème à cette alternative caricaturale : ou bien le dialecte est naturel, ou bien il n'est qu'un artefact du linguiste :

"A mon sens, aucun groupe de dialectes, de quelque façon qu'il soit formé, ne saurait constituer une famille naturelle, par la raison que le dialecte (qui représente l'espèce) n'est lui-même qu'une conception assez arbitraire de notre esprit/.../Il s'ensuit que le dialecte est une espèce bien plutôt artificielle que naturelle; que toute définition du dialecte est une definitio nominis et non une definitio rei ${ }^{\prime}$.

Entre l'ordre des choses et celui de la science, P. Meyer annule le sujet parlant et sa conception propre de son propre langage, ce que Ch. de Tourtoulon lui reprochera encore quinze ans plus tard :

"L'unité, l'individu à classer en matière de langage, c'est le parler de chaque homme. Nous avons tous une langue apprise dès le berceau, dans laquelle nous pensons, que nous employons dans l'intimité de préférence à toute autre. C'est ce langage naturel de chaque homme qui constitue l'unité linguistique ${ }^{2}$.

Cette dépossession des locuteurs s'oppose à ce que Vendryès pointera comme la "conscience linguistique », schème de perception pratique des limites relatives de l'intercompréhension et de la nature des différences entre dialectes. D'être polémique, cette déclaration est aussi intenable : on en verra les effets dans l'invention du terme "gallo-roman ", fiction géographique regroupant oc et oïl, et plus encore dans la fabulation savante concernant la « langue franke » de Lombardie (une scripta médiévale utilisée pour italianiser superficiellement des textes littéraires en ancien français) dont A. Mussafia et G. Paris prétendent établir les droits.

1. Paul Meyer, «Réponse ", Romania, 4, juillet 1875, p. 293-294.

2. Charles de Tourtoulon, Des dialectes, de leur classification et de leur délimitation géographique, Paris, Maisonneuve, 1890, p. 18. 


\section{Les dialectes au passé : la linguistique contre les régions}

Les romanistes parisiens dénient aux patois toute prétention littéraire : à cet égard, l'opposition entre la Revue des langues romanes, proche du Félibrige, et les directeurs de Romania, est tranchée. En témoigne l'accueil enthousiaste réservé aux premiers mémoires d'enquêtes dialectologiques, ceux de J. Cornu et J. Gilliéron, soutenus à l'EPHE en 1877. Désormais, les dialectologues se refusent à unifier des parlers qu'ils ne saisissent plus qu'au niveau de la commune, atomisant réalisations phonétiques et registres lexicaux, désavouant l'homogénéisation scripturale que privilégieraient des producteurs, écrivains ou érudits, pour une langue en procès de légitimation.

Dans cette dispersion, achevée par la dialectologie, de la forme des langues - et qui n'est que l'achèvement, du côté de la science, de la disqualification des parlers régionaux par le français -, la notion de dialecte ne reste opératoire que pour le plus lointain passé, et, par anticipation, comme le francien avait déjà réduit ses rivaux, la disparition des idiomes gallo-romans est déjà inscrite dans cette dénomination : « patois ». Encore restait-il à trouver quelque attestation de ce dialecte de l'Ile-de-France...

\section{La quête des textes}

Puisqu'un dialecte doit exister en Ile-de-France, il convient de le présenter dans ses attestations écrites, qu'elles relèvent : des témoignages de chroniqueurs ou de lettrés du Moyen Age; des documents d'archives qui en trahissent l'usage, ou du moins la présence (chartes, terriers, comptes, capitulaires...); de la littérature, populaire (contes, fabliaux, homélies...) ou non.

\section{Les témoignages}

Ils sont rarissimes : l'on ressasse celui de Francis Bacon ${ }^{1}$ et quatre ou cinq autres textes. Celui-là, de Conon de Béthune est le plus célèbre, introduit par Ampère, commenté par F. Génin et

1. Francis Bacon, Opus majus, III, 44 : "Nam et idiomata variantur ejusdem linguae apud diversos, sicut patet de lingua gallicana, quia apud Gallicos et Normannos et Picardos et Burgundos multiplici variatur idiomate». 
Guessard, il est repris, avant 1900, par Littré, G. Paris, A. Darmesteter et F. Brunot, entre autres :

« La roïne n'a pas fait que cortoise

Qui me reprist, ele et ses fius, li rois.

Encor ne soit me parole franchoise,

Si le puet on bien entendre en franchois

$\mathrm{Ne}$ chil ne sont bien apris ne cortois,

Qui m'ont repris, se j'ai dit mos d'Artois

Car je ne fui pas noris à Pontoise ».
La reine n'a pas agi " courtoisement »

Qui me reprit, avec son fils le roi.

Bien que ma parole ne soit pas d'Ile- de-France,

On peut ainsi la comprendre en français.

Et ceux-ci ne sont ni bien élevés ni courtois,

Qui m'ont repris quand j'ai usé de motsd'Artois ;

Car je n'ai pas été élevé à Pontoise.

\section{Les archives}

Les transcriptions du parler populaire, les textes les plus humbles des archives devraient recouper ces notations éparses, quelque peu infirmées par l'intellectualisation littéraire qui les exagère. Que le prix triennal Bordin, de l'Académie des Inscriptions et BellesLettres, réservé à des travaux philologiques, soit en 1880 libellé ainsi, en dit long sur les intentions des maîtres de l'école française de romanistique :

"Etudier à l'aide des documents d'archive et de textes littéraires le dialecte parlé à Paris et dans l'lle-de-France jusqu'à l'avènement des Valois. Comparer ce dialecte, d'après les résultats obtenus, à la langue française littéraire, et rechercher jusqu'à quel point le dialecte parisien était considéré au moyen-âge comme la langue littéraire de la France ».

En 1883, aucun essai n'ayant concouru, l'échéance du prix est reportée à 1885, sans plus de succès. Le sujet est abandonné, remplacé par l'étude des chroniques normandes et des noms de saints en France. L'échec est patent, mais F. Brunot, vingt ans plus tard, refuse encore de le reconnaître, écrivant : «Est-il d'autre part interdit de supposer que, si les documents manquent pour la région centrale et proprement française, c'est qu'ils ont disparu ou qu'on ne les a pas mis au jour ?/.../Au demeurant, ces questions de priorité sont assez vaines »'. p. 360 .

1. F. Brunot, Histoire de la langue française, Paris, A. Colin, 1966, tome 1, 
Qu'opposer à cet argument d'autorité du titulaire de la chaire d'histoire de la langue française à la Sorbonne ? S'il n'y a rien même à prix d'or -, cela signifie que les textes n'ont pas été assez cherchés, ou qu'ils ont disparu, mais, de toute façon, ça n'a aucune importance ! Quelle dénégation concernant une question dont l'enjeu n'est devenu décisif que par les investissements scientifiques (et sociaux) de ceux qui, désavoués par leurs propres recherches, affectent à présent d'en réduire la portée.

\section{La littérature}

Peu de témoignages, pas de documents : reste la littérature d'avant le $14 \mathrm{e}$ siècle. G. Fallot étayait sa thèse sur les Sermons de Maurice de Sully, le Partonopeus de Blois et Thibaut de Marly ; ses continuateurs n'en citent plus jusqu'à l'Histoire poétique de Charlemagne, de G. Paris, inspirée de la mythologie comparée de Max Müller. Deux hypothèses y sont avancées : d'une part, le cycle de Charlemagne (notamment le pèlerinage à Jérusalem) serait une transposition de certains des mythes fondateurs de l'humanité ; d'autre part, ces récits auraient reçu leur formulation poétique des «trouveurs » de la foire du Lendit, qui, rayonnant à partir de l'abbaye de Saint-Denis, l'auraient diffusé par toute la France.

Pour G. Paris, en dépit de tout ce qui a dû être perdu, la littérature de l'Ile-de-France comprend déjà, outre le Pèlerinage, Fierabras, les «branches les plus anciennes et les plus remarquables" du Roman de renart et neuf autres textes. Aucune analyse philologique n'étaie ces affirmations : l'exigence d'un dialecte de l'Ile-de-France, nécessaire aux reconstructions d'une grammaire historique du français, subordonne des textes, dont les versions connues sont récentes ou peu marquées dialectalement, à une cause nécessaire. L'exemple le plus flagrant en est l'adjonction de la Chanson de Roland après la défaite de 1870, G.Paris reconnaissant là une épopée bretonne, écrite en dialecte de l'Ilede-France, dont ne subsisteraient plus que des manuscrits normands.

En 1874, dans sa série des Epopées françaises, un des adversaires de G. Paris et P. Meyer, Léon Gautier, professeur à l'Ecole des Chartes, catholique, conservateur et partisan d'une science nationale, entreprend de réfuter les conjectures de G. Paris, spécialement sur la Chanson de Roland. C'est à un jeune élève de 
G. Paris, Charles Joret, qu'est dévolue, dans Romania (juillet 1874), puis dans les Mémoires de la Société de linguistique de Paris (1877), la charge de contrer L. Gautier par des arguments linguistiques. C. Joret, spécialiste des parlers normands, recourt à une démonstration phonétique à laquelle Gautier, plus historien que linguiste ne peut répondre, cependant que G. Paris entend se justifier par une nouvelle classification, à prétention sociologique :

"L'épopée nationale, née loin des villes et toute pénétrée de l'inspiration âpre et belliqueuse de la féodalité, devait subir une réfraction toute particulière en pénétrant dans un milieu aussi différent. C'est probablement dans les hautes sphères de ce monde parisien, sous l'influence directe de la royauté, que la chanson de Roncevaux a pris la forme qui nous est parvenue ; en face de cette poésie chevaleresque, le Pèlerinage de Charlemagne me paraît représenter la poésie bourgeoise : le premier de ces poèmes a dû plaire, comme on aurait dit bien plus tard, à la cour, le second surtout à la ville »'.

Ce détour explicatif réassure à $G$. Paris une interprétation disqualifiante - pour L. Gautier au moins - sur cette question. La conception de l'œuvre littéraire comme reflet des rapports sociaux emporte l'adhésion des cénacles d'intellectuels républicains qui retrouvent, dans l'enchantement des origines, une vénérable justification à leur patriotisme et une légitimité ancienne à leur déférence culturelle. Entre les remarques historiques érudites, pointilleuses de L. Gautier et les thèses emportées, brillantes, flatteuses surtout, de G. Paris, le débat est pratiquement (mais non scientifiquement) tranché, d'autant que celui-ci jouit d'une réputation méritée de savant. Plus tard encore, il inventera une géographie littéraire médiévale, réservant l'épopée au francien et au normand, les chroniques au picard et les récits didactiques et moraux au champenois.

Au total, tous les auteurs du $19 \mathrm{e}$ siècle recensent de zéro à trois ouvrages qui attesteraient le dialecte de l'Ile-de-France, sauf G. Paris qui n'en citera jamais moins de douze. A défaut de textes irréfutables, qui demeurent introuvables, il aurait fallu apporter le témoignage vivant d'un patois.

1. G. Paris, La poésie du moyen-âge, Paris, Hachette, 1885 (le texte cité est de 1877). 


\section{Enquête en Ile-de-France}

Constituer le parler parisien du Moyen Age comme l'origine du français moderne désavouait par avance toute enquête dialectale en Ile-de-France, sauf à considérer que la variation sociale vaut témoignage, dans les usages populaires, d'un état archaïque. Cette hypothèse est reprise, avec des sollicitudes variées, à propos de l'origine de l'argot ou du parler des valets de comédie. Rapidement, il est acquis qu'aucun secours n'en peut être attendu pour élucider la question du francien.

Quant à un parler rural vivant, représentant l'évolution naturelle, c'est-à-dire rurale, du francien, $A$. de Chevallet, en 1853, en soutient l'hypothèse qu'Arsène Darmesteter reprendra sans pouvoir l'exemplifier. Le raisonnement devient tautologique : aucune enquête ne peut attester de ce patois qui doit avoir déjà disparu puisqu'il n'est pas attesté, comme le déclare G. Paris : « Pour plusieurs de nos parlers provinciaux, pour ceux surtout qui vivaient à l'ombre redoutable de Paris, il est déjà trop tard; nous ne saurons jamais quelle forme spontanée aurait prise, dans les régions voisines de la capitale, le latin livré à lui-même " '.

Selon W. von Wartburg, quatre lexiques patois concernant l'Ilede-France ont paru entre 1833 et 1930 , soit un total de quaranteneuf pages (dont dix pour une nomenclature botanique). Pour son Atlas, J. Gilliéron affecte trois points (sur sept cents) à la région parisienne.Il n'est donc guère possible de restituer l'état dialectal de l'Ile-de-France il y a un siècle : on peut admettre une profonde déstructuration des patois par le français. Pourtant, un article sollicite l'attention: dans « Le patois de Sainte-Jamme (Seine-et-Oise) », Paul Passy, étudiant le parler de ses coreligionnaires et de sa domesticité, note que la ressemblance de ce patois avec le français expliquerait sa résistance (alors que tous les auteurs, à commencer par Littré, voient dans cette proximité la première raison d'une disparition) :

«Les villages de la vallée du ru de Gally, petit affluent de la Maudre, ont conservé jusqu'à ce jour un parler assez différent du parisien et du français d'école. Les différences, à vrai dire, ne sont pas assez marquées pour le rendre difficile à comprendre ; c'est probablement à

1. G. Paris, «Les parlers de France », Revue des Patois gallo-romans, 2, 1888, p. 161-175. 
cause de cela que ce parler a pu se conserver si longtems (sic), tandis que bien des patois plus différents ont complètement disparu "'.

P. Passy ajoute que les marques spécifiques de ce dialecte ne peuvent être reconstruites indépendamment d'une affectation sociale, incluant les paramètres d'âge, de sexe, de milieu, de formation scolaire, d'identité religieuse, de trajectoire, d'interactions et même d'aspirations sociales. Confronté à un parler nié par les romanistes, affranchi de leurs postulats, il retrouve le caractère intrinsèque de la variation sociale: n'ayant pas à reconstruire de formes « représentatives» qui, sur une carte, figureront la somme réglée des différences distinctives - comme le fera J. Gilliéron -, il démontre, dans le foisonnement empirique des faits, l'existence d'un improbable dialecte rural en Ile-deFrance.

\section{Le «francien »}

Au-delà du silence des textes, du mutisme des paysans, l'idée de dialectes contemporains, vivants, est refusée par les romanistes qui justifient les frontières de la France par l'unité gallo-romane. Le dialecte est un état dépassé du français. Pas d'étude dialectologique qui ne comprenne un préambule sur la disparition trop rapide des patois, sans qu'on puisse distinguer entre la description élégiaque et la prophétie autorisée, c'est-à-dire l'anticipation savante qui crédibilise l'inéluctabilité du processus. Parce que le danger fédéraliste un instant entrevu se dissipe, parce que l'agonie de ces idiomes est sûre, les romanistes, ayant imposé une définition légitime des patois qui interdit leur résurrection ou leur sécession, entendent s'assurer le monopole de la dialectologie.

En 1887, deux revues sont créées simultanément, la Revue des patois de Léon Clédat et la Revue des patois gallo-romans de J. Gilliéron et Rousselot. La même année, une chaire de «langues et littératures picarde et wallonne » est fondée à Lille, et, surtout, G. Paris prononce son fameux discours sur les "Parlers de France ", un appel largement médiatisé à destination de la petite intelligentsia (instituteurs, curés, notables communaux), convoquée pour un travail linguistique de terrain. L'ambition et les limites

1. P. Passy, «Patois de Sainte-Jamme (Seine et Oise)", Revue des patois gallo-romans, IV, 13, 1891, p. 7-16. 
en sont fixées par G. Paris qui se réclame de P. Meyer (contre G.-I. Ascoli) et, effaçant ce que le caractère polémique de l'argumentation emportait de conjoncturel et de radical, en transfigure la conclusion en loi.

« En faisant autour d'un point central une vaste chaîne de gens dont chacun comprendrait son voisin de droite et son voisin de gauche, on arriverait à couvrir toute la France d'une étoile dont on pourrait de même relier les rayons par des chaînes transversales continues. Cette observation bien simple, que chacun peut vérifier, est d'une importance capitale; elle a permis à mon savant confrère et ami, M. Paul Meyer, de formuler une loi qui, toute négative qu'elle soit en apparence, est singulièrement féconde, et doit renouveler toutes les méthodes dialectologiques: cette loi, c'est que, dans une masse linguistique de la même origine que la nôtre, il n'y a réellement pas de dialectes "'.

L'incidence de ces propos dépend de l'interprétation donnée à « réellement » - quelle est la réalité d'un fait de langue qui exclut le jugement du locuteur? - et de ce que désigne " une masse linguistique de la même origine que la nôtre " - s'agit-il du "gallo-roman» ou de la Romania toute entière ? G. Paris ne tranche pas, s'en tenant à l'unité de la langue littéraire comme exclusive des dialectes. Cette unité n'étant pas accomplie au Moyen Age, les textes mentionnés par G. Paris - mais refusés par L.Gautier - devraient prouver qu'il existait un dialecte particulier à l'Ile-de-France.

Prenant prétexte de l'ouvrage de $\mathrm{H}$. Suchier ${ }^{2}$, G. Paris impose en 1889 le nom de «francien » qui ne correspond ni à une désignation, ni même à une province du moyen-âge puisque, à peu près au même moment, A. Longnon démontre que l'Ile-deFrance est une création tardive du pouvoir royal, coalescence de fiefs disparates réalisée au $16 \mathrm{e}$ siècle.

A partir de 1890 , le terme de francien est admis mais son usage reste marginal. Dans Origine et structure de la langue française, W. von Wartburg l'emploie une seule fois et, d'un survol des grammaires historiques du français, ressort l'impression d'un évitement généralisé. Faute de mieux, semble dire le linguiste.

De fait, "francien " occupe, et justifie a posteriori, cette antécédence du français que les romanistes devaient supposer, pour des raisons de configuration épistémologique et de représentation socio-historique, au principe de la langue que la République

1. G. Paris, "Les parlers de France ", art. cité, p. 163.

2. Cf., sur l'usage des travaux de Metzke et H. Suchier, l'article de J. Chaurand, spécialement les pages 92 à 94 . 
entreprend de diffuser par l'obligation scolaire. Incapables de produire une preuve irréfutable de son existence, les comparatistes en ressassent l'inéluctabilité historique et géographique comme en témoignerait ce schéma esquissé par G. Paris en 1897.

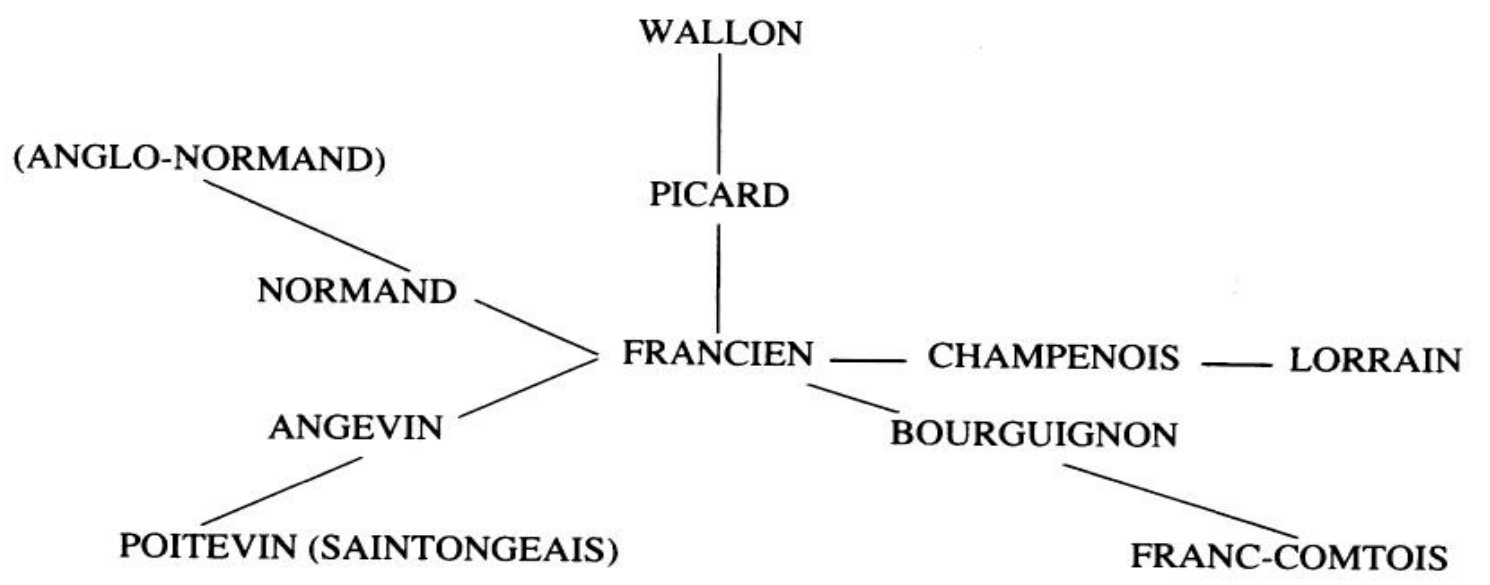

L'image d'une France naturellement rassemblée autour de sa capitale -qui reste en blanc sur les cartes dialectales- s'impose ; les patois ne sont plus que des écarts, destinés à se fondre dans le blanc, à « passer ». A l'image prégnante du réseau ferroviaire se superpose la disposition en rayons que choisira J.Gilliéron pour la numérotation des points de l'Atlas. Ce schéma spatial qui annule le temps et les gens, qui soumet à un centre idéal le foisonnement désordonné des langues, induit la présence d'un observateur qui domine et qui sait ; c'est J.Gilliéron dirigeant E. Edmont de son bureau parisien, G. Paris tançant les érudits départementaux réunis à la Sorbonne. Ce qui s'esquisse, c'est une conception jacobine de la science comme domination méritocratique de l'espace.

\section{Le recours métaphorique}

Si la validation du concept de francien, suspendue devant la carence des textes et des enquêtes, dépend de la position institutionnelle de ceux qui le nomment, sa formulation, pour prendre consistance, doit recourir à une métaphorisation, identiquement déclinée par les tenants de thèses opposées, mêlant la géographie à la flore et à la vision colorée. 


\section{La géographie (G. Fallot, A. de Chevallet, J. Gilliéron)}

Du discours qu'il invente - la dialectologie historique du français -, G. Fallot cherche à construire économiquement la logique en jouant sur deux métaphores : l'une, secondaire, qui décrirait la transition géographique entre les parlers et le registre chromatique, s'impose ; l'autre, essentielle, qui expliquerait les différences par la stratification chronologique, transformée par les contacts, les lieux, les locuteurs, et c'est la géomorphologie qui domine : «Je les nommerai/les dialectes/, non point du nom d'une province dans laquelle ils fussent exclusivement parlés, mais du nom de celle dans le langage de laquelle leurs caractères se trouvent le plus saillants, le mieux réunis et le plus complètement en relief, normand, picard et bourguignon ${ }^{1}$.

Le contexte des ruines romantiques et des révolutions du globe de Cuvier est proche. A. de Chevallet, un adversaire de G. Fallot, y recourt pour postuler une composante gauloise du français, transformant la métaphore géologique en conjonction d'une langue et d'un sol : «De même que les naturalistes reconnaissent les catastrophes du globe dans les différentes couches de terre, de rochers et d'argile; de même un esprit analytique parviendrait à distinguer dans la langue d'un peuple les différentes couches de langues étrangères qui constatent les catastrophes des empires ${ }^{2}$. Longtemps, les romanistes sépareront l'approche philologique, fondée sur la diachronie et le recollement des écritures, et l'enquête de terrain; il faut attendre la géographie linguistique de J. Gilliéron pour que soit métaphoriquement ressoudées ces deux approches.

\section{La botanique (F. Génin, Darmesteter)}

L'image de la flore, d'une vie organique des patois, apparaît chez F. Génin comme la réponse à ce qu'il dénonce comme les " édifices » et les « constructions» artificielles de G. Fallot et de la science allemande. Cette métaphore suit un cheminement latéral, à peine perceptible dans certaines occurrences - comme "culture " chez Littré ou «inculte» chez $A$. de Chevallet - qui réfèrent indécidablement au culturel et à l'agricole. C. Aubertin, un vulgarisateur sévèrement critiqué par les romanistes, renoue de

1. G. Fallot, op. cit., p. 14.

2. A. de Chevallet, Origine et formation de la langue française, Paris, Imprimerie impériale, 1853, p.XII. 
manière systématique avec ce registre que la jeune école comparatiste reprend, sans indication de source. A. Darmesteter en propose cette relecture enrichie par les références darwiniennes : "C'est ainsi que le latin populaire, semé par la conquête romaine sur le sol de la Gaule, la couvrit d'une immense flore linguistique, qui prit en se développant en tous sens, à l'infini, des aspects infiniment variés ${ }^{\prime}$.

Associant métonymiquement la fleur à ses couleurs, le terme « nuance " s'impose à Darmesteter. En un mot, en faveur de la thèse de dialectes distincts, avec des frontières tranchées, les linguistes recourent au modèle géologique; contre, ils arguent des transitions chromatiques de la palette. La fleur, enracinée dans le sol mais épanouissant ses couleurs au soleil, représente un moyen terme, disputé par les deux camps.

\section{La tapisserie (G. Paris)}

G. Paris métaphorise ainsi la dialectologie : «Mais si nous ne pouvons empêcher la flore naturelle de nos champs de périr devant la culture qui la remplace, nous devons, avant qu'elle disparaisse tout à fait, en recueillir avec soin les échantillons, les décrire, les disséquer et les classer pieusement dans un grand herbier national $»^{2}$.

De ce texte, auquel il est fait constamment référence, on omet l'essentiel. G. Paris ne se contente pas d'abstraire les patois de leurs locuteurs et de prophétiser leur mort linguistique afin de les embaumer philologiquement, il renoue surtout, en préférant le chauvinisme de F. Génin au darwinisme de Darmesteter, avec la métaphore d'une affinité entre la terre et la langue, qu'il aurait récusée vingt ans plus tôt, quand il argumentait contre les prétentions pangermanistes. Les déracinés de $M$. Barrès n'appartiennent pas à une autre représentation. Cette vision suspend l'explication : il n'y a plus d'histoire à déployer, seulement un étiquetage minutieux que la petite intelligentsia peut exécuter. Le résultat est livré par avance dans un lyrisme discrètement annoncé par « nuance»:

"Cette muraille imaginaire, la science, aujourd'hui mieux armée, la renverse, et nous apprend qu'il n'y a pas deux Frances, qu'aucune

1. A. Darmesteter, Cours de grammaire historique, Paris, Delagrave, 1890 , tome 1, p. 30.

2. G. Paris, «Les parlers de France », art. cité, p. 168. 
limite réelle ne sépare les Français du nord de ceux du midi, et que d'un bout à l'autre du sol national nos parlers populaires étendent une vaste tapisserie dont les couleurs variées se fondent sur tous les points en nuances insensiblement dégradées "'.

On ne s'étonnait pas qu'un romaniste célèbre, visant l'Académie Française, ait préféré l'art éminemment médiéval de la tapisserie à une référence picturale impressionniste, qui eût été mieux trouvée, mais probablement incomprise de l'auditoire. On ne s'étonnera plus que le savant cherche une caution dans « la science » et l'éclipse aussitôt par l'éclat de sa métaphore.

Un dernier mot sur l'émergence, la disparition et la résurgence des métaphores : prégnantes entre 1829 et 1855 , lors de l'élaboration du discours dialectologique, elles s'évanouissent entre 1855 et 1885, lorsque l'existence du dialecte de l'Ile-de-France paraît assurée (Littré n'en fait aucun usage). Quand la controverse risque d'être ravivée par l'absence de textes irréfutables, la prolifération métaphorique compense ces lacunes, emportant une conviction qui, hors de la cohérence des images, n'a plus de consistance qu'imaginaire.

\section{Conclusion}

La fonction de «francien » est donc d'opérer l'amnésie collective sur le caractère fictif de son existence et sur l'arbitraire de son empire sur les patois. Figeant l'histoire de la langue française en une formule qui dure, "un patois qui a réussi », les romanistes dissimulent, sous l'humilité apparente de la maxime, leur affirmation quant à la réalité d'un dialecte de l'Ile-de-France. L'intention finaliste transparaît immédiatement : la France devient une entité pré-construite par la civilisation gallo-romane; Paris, sa capitale naturelle; le français, sa langue élue par le concours d'un peuple. Sans recourir à la stigmatisation, les romanistes annoncent et justifient l'exclusion des patois de toute légitimité particulièrement de l'écriture-, confortant les cercles dominants, politiquement et culturellement, dans leur politique linguistique au nom de la précellence, littéraire et historique, du français.

L'enjeu n'aurait pas eu ce caractère passionné sans l'orgueil blessé du nationalisme, défendant le francien comme les républi-

1. Ibid., p. 164. 
cains défendaient Paris en 1870, soutenant le droit d'un patois moins entaché de germanisme que n'apparaissaient les dialectes bourguignon, picard ou normand. Et, dans l'enthousiasme de G. Paris, tissant idéalement l'unité de la France -comment ne penserait-on pas aux Tapisseries de Péguy ?-, ne faut-il pas voir l'exaltation d'une homonymie, même si G. Paris, successeur de son père dans la chaire de Langue et littérature françaises du Moyen Age au Collège de France, est assuré de ses origines.

« Mais toute culture est lourdement chargée de symbolisme, comme tout comportement, même le plus simple; le comportement est moins fonctionnel qu'il n'y paraît ; il obéit à des impulsions inconscientes et même inavouées; le comportement est symbole. Les raisons données rationalisent a posteriori un comportement qui se trouve à la merci de l'inconscient. Même une théorie scientifique complexe et documentée n'est parfois qu'un symbole des besoins inconnus du moi. Si le savant milite en faveur de ces théories, ce n'est pas qu'il les croie vraies, c'est qu'il les voudrait telles ${ }^{1} »$.

1. E. Sapir, Anthropologie, Paris, Minuit, 1968, p. 53. 


\section{Résumé /Abstract / Compendio}

\section{LE FRANCIEN (1815-1914): LA LINGUISTIQUE AU SERVICE DE LA PATRIE}

Le francien, dialecte de l'Ile-de-France, représenterait le français « standard » du Moyen Age, mais ce concept correspond en fait à une attente jacobine des classes dominantes de la Troisième République, relayée par des universitaires en quête de légitimité, et à une stabilisation du marché de la linguistique sous la férule de G. Paris. Outil opératoire et symptôme d'une politique de la science vouée à une histoire apologétique du français, le « francien » relève de la fiction scientifique.

Mots-clés : Dialectologie française, francien, Histoire de la linguistique (19e siècle)

THE «FRANCIEN : LINGUISTICS IN THE SERVICE OF HOMELAND

The "francien " is the dialect of Ile-de-France, it would have represented the standard French in the Middle Ages, but, in fact, this concept corresponds to the jacobin views of the Third Republic ruling classes, converging with university scholars longing for social recognition, new wording and linguistic stabilization. Devoted to the praise of the french language, the "francien " seems to be a scientific fiction.

Key-words : French dialectology, «francien », History of linguistics (19th century)

\section{EL *FRANCIEN", LINGUISTICA AL SERVICIO DE LA PATRIA}

El *francien », dialecto de l'Ile-de-France, representaría el francés "standard " de la Edad Media, pero de hecho este concepto corresponde a una esperanza jacobina de las clases dominantes de la Tercera República, retomada por unos universitarios en busca de una legitimidad y de una estabilización del mercado de la lingǘstica bajo la férula de G. Paris. Instrumento operativo y sintoma de una política de la ciencia dedicada a una historia apologética del francés, el *francien " parece ser una ficción cientifica.

Palabras-claves : dialectología francesa, "francien », historia de la lingüística $\left(19^{\circ}\right.$ siglo) 